\title{
Nanoscale 3D Refractive Indices Mapping on Native Cheek Cells by Axial Scanning Transmission Electron Tomography.
}

\author{
Yue $\mathrm{Li}^{1}$, Jinsong $\mathrm{Wu}^{3}$, Reiner Bleher ${ }^{3}$, Vinayak Dravid ${ }^{3}$, and Vadim Backman ${ }^{2}$ \\ ${ }^{1}$ Applied Physics Program, Northwestern University, Evanston, Illinois 60208, USA. \\ 2. Department of Biomedical Engineering, Northwestern University, Evanston, Illinois 60208, USA. \\ ${ }^{3}$.Department of Materials Science and Engineering, Northwestern University, Evanston, Illinois 60208, \\ USA.
}

Scanning transmission electron microscopy (STEM) has been engaged in the study of ultrastructure of biological samples both qualitatively and quantitatively since the 1970s[1] .Equipped with secondary electron (SE) detector, axial (TE) detector, and annular dark field (ZE) detector, STEM is able to image with secondary electron contrast, phase contrast, as well as mass-thickness contrast of the same region easily. In particular, high angle annular dark field (HAADF) detector collects electrons been scattered above a pre-defined large angle. The contrast given by those high-angle scattered electrons, which are described by Rutherford's scattering model, is proportional to mass. Thus nano-scale, quantitative 2D projection mass mapping of biological complex can be done by calibrating HAADF images with molecules with similar chemical composition and known mass or density[2]. However, 2D projection data is notoriously hard to interpret, if not impossible. To tackle the problem, 3D tomography information is needed. It has been proven that 3D tomography of a whole human erythrocyte infected with the malaria parasite Plasmodium falciparum can be reconstructed by tilting each of the thick serial cryo-sections (around 1 $\mu \mathrm{m}$ )with 8-10 nmresolution using STEM axial detector[3].

Field carcinogenesis, also known as cancer field effect, has two most distinguishable characteristics: (a) It occurs prior to histological changes; (b) It's not restricted in areas that later develop into malignant tumors[4]. In clinical study, it is optical imaging and spectrometry that are widely applied, instead of time consuming and skill demanding electron microscopy. Subramanian et al. [5] in Backman's lab in Northwestern have successfully developed a fast, non-invasive method named Partial Wave Spectrometry (PWS) to detect field carcinogenesis in colon cancer and lung cancer. The resolution of PWS is around 50 to $100 \mathrm{~nm}$, which is far beyond the $200 \mathrm{~nm}$ diffraction limit of light. To validate PWS, we need a 3D refractive index map of the entire cell. For biological samples, the local refractive index is linear to the local density [6]. So by measuring mass distribution and topographic reconstruction, we can calculate refractive index accordingly.

To achieve ultrahigh resolution, cells are usually sectioned to less than $300 \mathrm{~nm}$ thick for STEM, and even thinner for convention TEM However, in order to keep cells in the most native state, neither sectioning nor staining are acceptable. Maintaining the quality of information in native samples requires longer pixel dwell time thus require longer electron exposure, but radiation damage becomes significant with more time under the beam. There is a trade-off. For the purpose of field carcinogenesis detection, getting information from an intact cell dominates. Despite its importance, the micro-scale refractive index map of an entire cell in its native state has not been investigated.

In this study, we utilized the axial STEM imaging and tomography reconstruct a 3D refractive index map of a cheek cell in its native state. The cheeks cells are fixed by glutaraldehyde and formaldehyde then critical point dried to keep structure. Figure 1 shows TE-STEM images at $-60^{\circ}, 0^{\circ}$, and $60^{\circ}$. Since 
the intensity in TE mode for thick samples obeys Bill-Lambert Law [7], we then calibrate the intensity with mass using polystyrene beads. The alignment of STEM images is done by patch tracking algorithm and SIRT algorithm embedded in IMOD. After reconstruction, the intensity is converted to mass then to refractive index. Figure 2 shows the 3D structure of the nucleus and organelles after volume rendering and visualizing by Amira5.5. All STEM images were acquired using Hitachi HD-2300A STEM at room temperature with no significant beam damage occurred. Obtained mass distribution and refractive index map will be presented.

\section{References:}

[1]Engel, A. and C. Colliex, Application of scanning transmission electron microscopy to the study of biological structure. Current opinion in biotechnology, 1993. 4(4): p. 403-411.

[2] Pennycook, S., Z-contrast transmission electron microscopy: direct atomic imaging of materials. Annual review of materials science, 1992. 22(1): p. 171-195.

[3] Hohmann-Marriott, M.F., et al., Nanoscale 3D cellular imaging by axial scanning transmission electron tomography. Nature methods, 2009. 6(10): p. 729-731.

[4] Backman, V. and H.K. Roy, Advances in biophotonics detection of field carcinogenesis for colon cancer risk stratification. Journal of Cancer, 2013. 4(3): p. 251.

[5] Subramanian, H., et al., Nanoscale cellular changes in field carcinogenesis detected by partial wave spectroscopy. Cancer research, 2009. 69(13): p. 5357-5363.

[6] Barer, R. and S. Joseph, Refractometry of living cells part I. Basic principles. Quarterly Journal of Microscopical Science, 1954. 3(32): p. 399-423.

[7] Thomas, D., et al., Mass analysis of biological macromolecular complexes by STEM. Biology of the Cell, 1994. 80(2-3): p. 181-192.
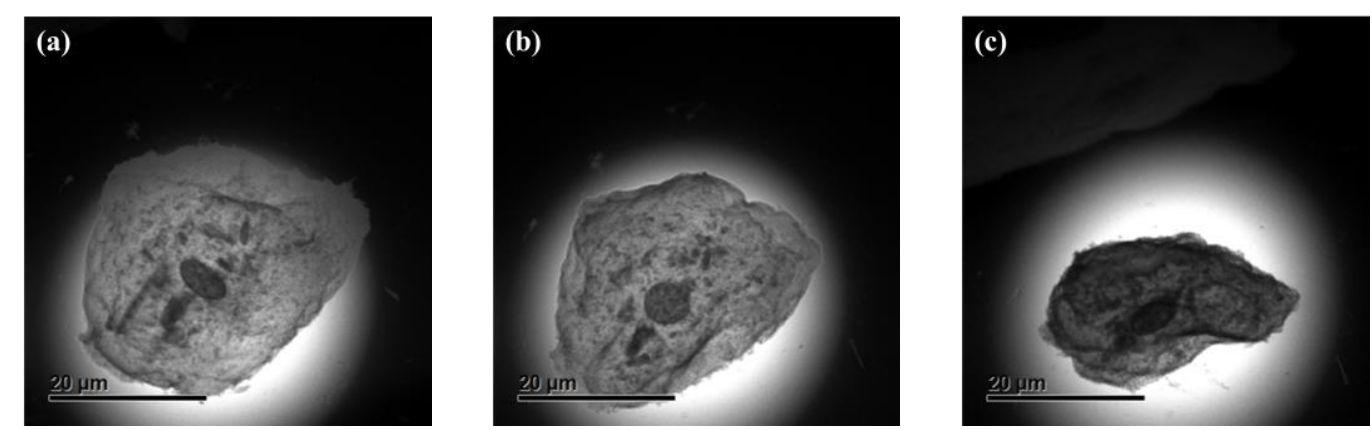

Figure 1.Axial-STEM images of an entire critical point dried cheek cell at (a) $-60^{\circ}$, (b) $0^{\circ}$ and (c) $60^{\circ}$.
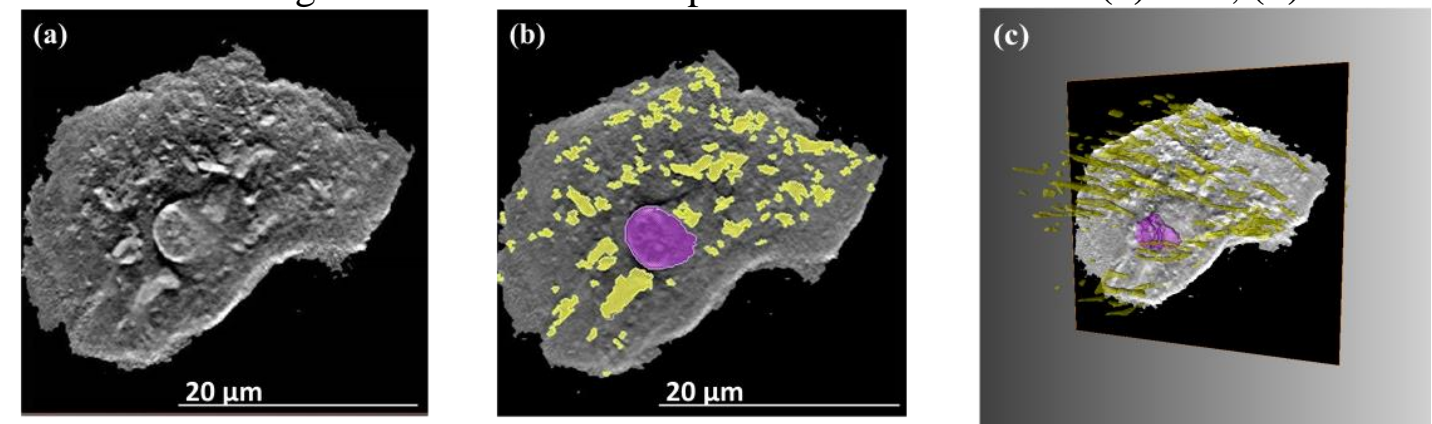

Figure 2. 3D reconstruction of the entire cheek cell. (a) virtual 2D slice in the middle of the cell, (b) segmentation of nucleus and organelles by mass, and (c) volume rendering of nucleus and organelles 